\title{
Exaggerated Mini Puberty of Early Infancy in the Preterm Girls: A Case Report and Systematic Review
}

mohamad Ahangar davoodi ( $\square$ m.davoodi@arakmu.ac.ir)

Arak University of Medical Sciences https://orcid.org/0000-0001-9569-7151

Fatemeh Ghobadi

Arak University of Medical Sciences

Golsa Mahmoudi

Arak University of Medical Sciences

Almasi-Hashiani Amir

Arak University of Medical Sciences

Research article

Keywords: Mini puberty, Infancy, Preterm, Gonadotrophins, Hypothalamic-pituitary-gonadal Axis, Sex Steroid, GnRH Agonist

Posted Date: June 17th, 2020

DOI: https://doi.org/10.21203/rs.3.rs-32210/v1

License: (a) (i) This work is licensed under a Creative Commons Attribution 4.0 International License. Read Full License 


\section{Abstract}

Background: Mini puberty is the important period of infancy life that has several impressions to sexual development in both sexes. In the infants born very premature and extremely low birth weight, mini puberty changes including clinical, hormonal and imaging data are severe and long lasting, especially in girls. It can be called "exaggerated mini puberty".

Methods: In this study, 6-month preterm infant with bronchopulmonary dysplasia presented with periodic vaginal bleeding, breast stage tanner 3 and pubic hairs. We followed up infant by clinical and para clinical evaluations during one year and compared this data with all of the similar articles were published.

Results: During one year monitoring of the infancy puberty presentation, gradually returned to the pre pubertal state, without excessive hormonal treatment.

Conclusions: Due to this systematic review, exaggerated mini puberty is the temporary physiologic state in very low birth weight preterm infants that should be prevents any extra hormonal and surgical intervention.

\section{Background}

Gonadotropin-releasing hormone secretion in 17-18 weeks of fetus' life causes an increase in Luteinizing hormone (LH) and Follicle-stimulating hormone (FSH) and then with the decrease in gonadotropins during the last months of embryonic, the reduction trends of sexual hormones are observed due to the fetal placenta estrogen negative feedback [1]. During the first months of life, temporary activation of the HPG axis (hypothalamus-pituitary gonad) occurs that was described in 1970 for the first time and called "mini puberty" [2, 3].

In male infant within the mini puberty, LH serum level is dominant and FSH serum level is dominant in female [4]. In both sexes LH levels ordinarily decrease by 6-9 months of age, but in females FSH levels could stay high, up to the age of 3-4 years [4,5]. Testosterone levels in boys start to increase after 1 week of age, and peak to pubertal levels at 1-3 months then decline to low pre pubertal levels by approximately 6 months of age [4]. Estradiol level is high at delivery for girls and decreases within the first week of life. It increases to $5-50 \mathrm{pg} / \mathrm{mL}$ between the days 30 to 60 and then reduces to about $15 \mathrm{pg} / \mathrm{mL}$ [6]. The estradiol fluctuation levels in girls are probably because of the growth and atrophy of the follicles and reaches the before puberty level till the age of 2 years but the peak of estradiol serum is at the age of 6 months [6].

In male infant androgen exposure of mini-puberty also predicts later sex-typed trait (brain muscularization), longitudinal good testicular function such as spermatogenesis, penile and testicular size, prostate growth $[4,5]$. In girls, uterine and breasts enlarge, but sebaceous gland and acne development and transient pubic hair are seen in both sexes $[4,6]$. In contrast to the boys, it is not known that the imprint of mini puberty in future development of woman sexual limbs, trait and reproductive systems [6].

Gonadotropins level, especially FSH, in preterm infants is higher than term newborns and stays high for a longer period of time, particularly in girls. In $38 \%$ of the studied preterm girls, FSH was higher than $4.5 \mathrm{IU} / \mathrm{L}$. In addition, higher levels of testosterone and faster growth of the testicles and penis are observed among the premature boys compared to term infants. Also FSH levels in small for gestational age (SGA) infants are higher than appropriate for gestational age (AGA) infants [6].

In congenital hypogonadotropic hypogonadism, both fetal and postnatal pituitary gonadotropin secretion is low, that present with micro phallus and undescended testis and absence of mini puberty changes in infancy $[4,7,8]$. The infant with 45,X (not another mosaicism) is higher FSH level than normal and remain elevated up to 6 years of age [9]. Lack of mini puberty presentations in the infancy are helpful to diagnosis some of disorder such as turner syndrome and congenital hypogonadotropic hypogonadism [6]. So mini puberty is golden time window to diagnose and treatment of congenital hypogonadotropic hypogonadism boys in infancy $[1,5,10,11]$.

In the infants with Difference in Sex Development (DSD) at the mini-puberty phase LH/FSH ratio of 0.32 is helpful to distinguish the infant boys from girls [5, 12]. In the mini-puberty ultrasonography data, the height of the uterus is $35 \mathrm{~mm}$ and the highest width is around $14 \mathrm{~mm}$. About $25 \%$ of the infants have a little fluid in the endometrial space. In $84 \%$ of the infants, follicles with diameters under $1 \mathrm{~cm}$ are observed [6]. In this study, we report a mini puberty case and also conducted a systematic review on mini puberty cases.

\section{Methods}

We present a 6-month preterm infant with periodic vaginal bleeding, breast stage tanner 3 and pubic hairs. Serum estradiol, LH and FSH were measured by using chemiluminescence assay (Simens instruments model Immulite XP2000, USA) method. All Ultrasonography finding were done and reported by an expert sonologist. The patient was followed by pediatric endocrinologist for one year. Given to confidentially, the parents agreed to participate through a written informed consent.

We systematically searched the three main international databases to fine the related published papers including Pubmed/Medline, Scopus and ISI/Clarivate Analytics. The searches were done from the inception to the April 2020 and all English full-text papers were included in this study.

The research strategy used the combination of some related keywords including; exaggerated mini puberty, mini puberty, very low birth weight, infancy, preterm and premature, hypothalamus-pituitary-gonadal axis, excessive ovarian stimulation syndrome, GnRH agonist agents and case report. Among retrieved records, 14 cases from 8 studies were included in this systematic review. Analysis was done by Stata software version 13. 
A six-month-old infant with the weight of 4100 grams, 55-centimeter height and head circumference of $37.5 \mathrm{~cm}$ visited the clinic due to symptoms of sexual prematurity including 2 periodic vaginal bleeding; breasts were at tanner stage 3 and pubic hair at stage 2 .

The infant was born from a preterm labor of 25 weeks of gestation, with a birth weight of 800 grams and the history of 50 -day NICU hospitalization. Because of Bronchopulmonary dysplasia and congenital hypothyroidism, she has been taking Levothyroxine $25 \mathrm{mcg}$ daily and also Hydrochlorothiazide and Furosemide, Atrovent and Flixotide oral inhalers, and she was oxygen dependent.

Central precocious puberty was obvious in laboratory evaluations including Estradiol $54.9 \mathrm{pg} / \mathrm{ml}, \mathrm{FSH} 4.1 \mathrm{mUl} / \mathrm{ml}$ and $\mathrm{LH} 5.3 \mathrm{mUl} / \mathrm{ml}$. Other lab results were normal except ferritin, which is reasonable considering the inflammatory process of the patient's "BPD" and became normal within an eight-month monitoring. Furthermore, these data were reported by sonography: Uterine size of $1.4 * 21 * 17$ millimeters $(\mathrm{mm})$ and volume of $2.1 \mathrm{milliliter}(\mathrm{cc})$, endometrial thickness of $4.4 \mathrm{~mm}$, cervical length of $18 \mathrm{~mm}$ and ovaries' diameters containing 1.3 and $3.5 \mathrm{~mm}$ with $6.6 \mathrm{~mm}, 8.8 \mathrm{~mm}$ and $10.5 \mathrm{~mm}$ follicles.

Hypothalamus and pituitary and its axis MRI with and without contrast were normal. By monthly assessments after two months the infant breast size got slightly smaller but it was still at stage-3, pubic hair disappeared but was continued menarche and the decrease in gonadotropins and estradiol levels were observed in the tests results (Table 1).

Table 1

The Infant puberty presentations; clinical, laboratory and radiology changes during the monitoring

\begin{tabular}{|c|c|c|c|c|c|c|}
\hline AGE & PRESENTATION & $\begin{array}{l}\text { LH } \\
(\mathrm{mlU} / \mathrm{ml})\end{array}$ & $\begin{array}{l}\text { FSH } \\
(\mathrm{mlU} / \mathrm{ml})\end{array}$ & $\begin{array}{l}\text { ESTRADIOL } \\
(\mathrm{pg} / \mathrm{ml})\end{array}$ & $\begin{array}{l}\text { FERRITIN } \\
\text { (ng/ml) }\end{array}$ & SONOGRAPHY \\
\hline 6 & VB+ & 5.3 & 4.1 & 54.9 & 1118 & Utreus: $21 \times 11.4 \times 17 \mathrm{~mm}=2.1 \mathrm{cc}$ \\
\hline \multirow[t]{3}{*}{ MONTH } & B3 & & & & & Endometrium: $4.4 \mathrm{~mm}$ \\
\hline & $\mathrm{PH}: 2$ & & & & & Ovaries $\left\{\begin{array}{c}L: 17 \times 10.5 \times 14 \mathrm{~mm}=1.35 \mathrm{cc} \\
R: 24 \times 18 \times 14.5 \mathrm{~mm}=3.5 \mathrm{cc}\end{array}\right.$ \\
\hline & & & & & & Follicles: 10.5, 8.8, $3.5 \mathrm{~mm}$ \\
\hline \multirow[t]{3}{*}{7 MONTH } & VB+ & 5.01 & 4.4 & 29.3 & 568 & \\
\hline & $\mathrm{B}: 3$ & & & & & \\
\hline & $\mathrm{PH}: 2$ & & & & & \\
\hline \multirow[t]{3}{*}{$8 \mathrm{MONTH}$} & VB+ & 1.21 & 2.79 & 20.28 & & \\
\hline & $B: 3$ & & & & & \\
\hline & $\mathrm{PH}: 1$ & & & & & \\
\hline \multirow[t]{3}{*}{9 MONTH } & VB+(spotting) & 0.79 & 2.11 & 14.23 & 187.7 & Uterus: $23 \times 11 \times 11.5 \mathrm{~mm}=2 \mathrm{cc}$ \\
\hline & $\mathrm{B}: 2$ & & & & & Ovaries $\left\{\begin{array}{l}L: 15 \times 6.5 \times 13 \mathrm{~mm}=0.7 c c \\
R: 18 \times 10 \times 18 \mathrm{~mm}=1.7 c c\end{array}\right.$ \\
\hline & & & & & & Follicles: $6.5,6,5.5 \mathrm{~mm}$ \\
\hline \multirow[t]{3}{*}{$13 \mathrm{MONTH}$} & B:bud & 0.65 & 2.9 & 30.68 & 51.68 & Uterus: $29 \times 15 \times 7 \mathrm{~mm}=1.8 \mathrm{cc}$ \\
\hline & & & & & & Ovaries $\left\{\begin{array}{c}L: 17 \times 13.5 \times 7.5 \mathrm{~mm}=0.9 c c \\
R: 18 \times 12 \times 8 \mathrm{~mm}=0.9 c c\end{array}\right.$ \\
\hline & & & & & & Follicles: $8.5,9 \mathrm{~mm}$ \\
\hline 18 MONTH & Pre pubertal & 0.08 & 1.63 & 8.31 & 50.16 & \\
\hline
\end{tabular}


Table 1

The review of all reported mini puberty of early infancy in the preterm girls

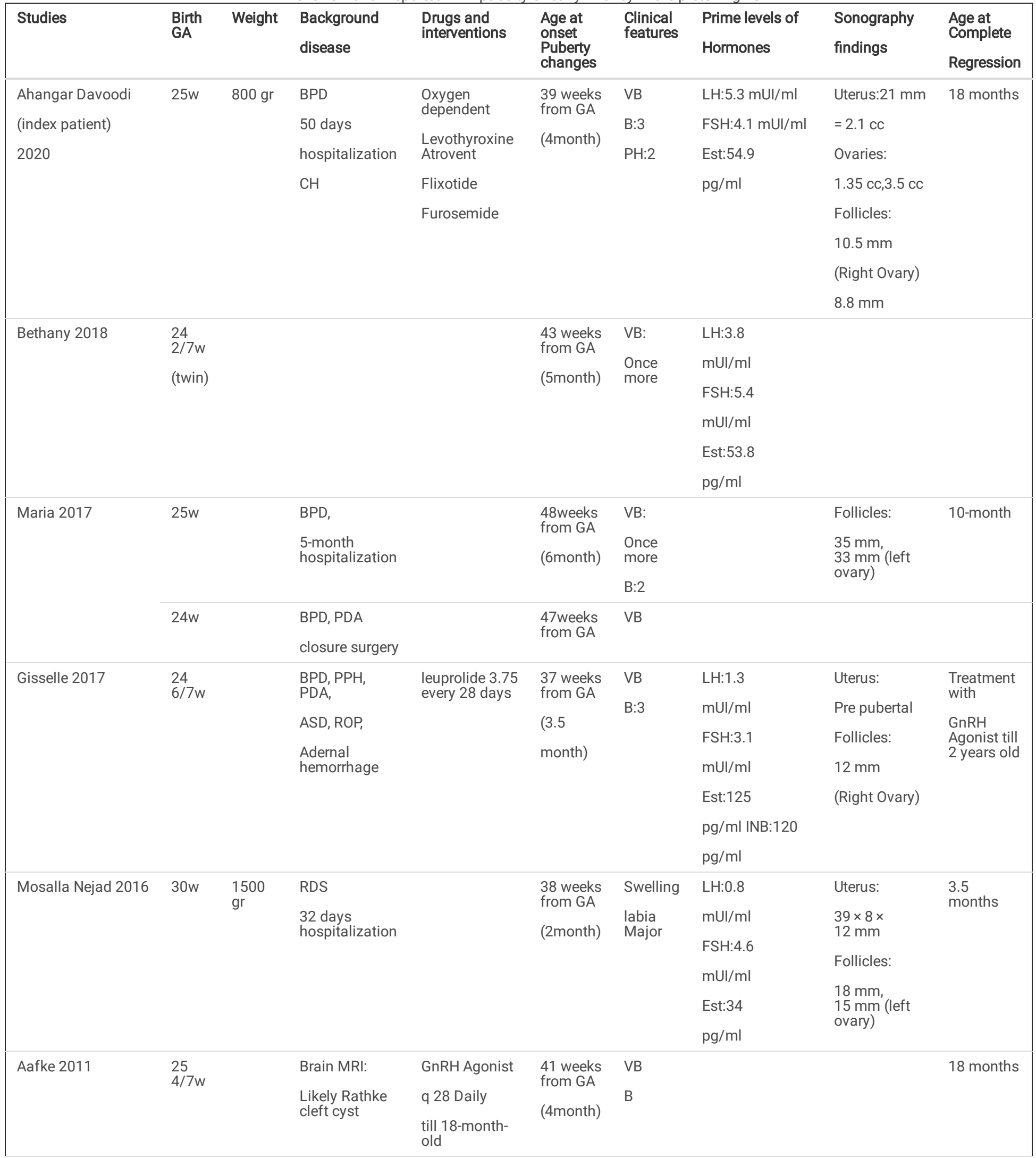




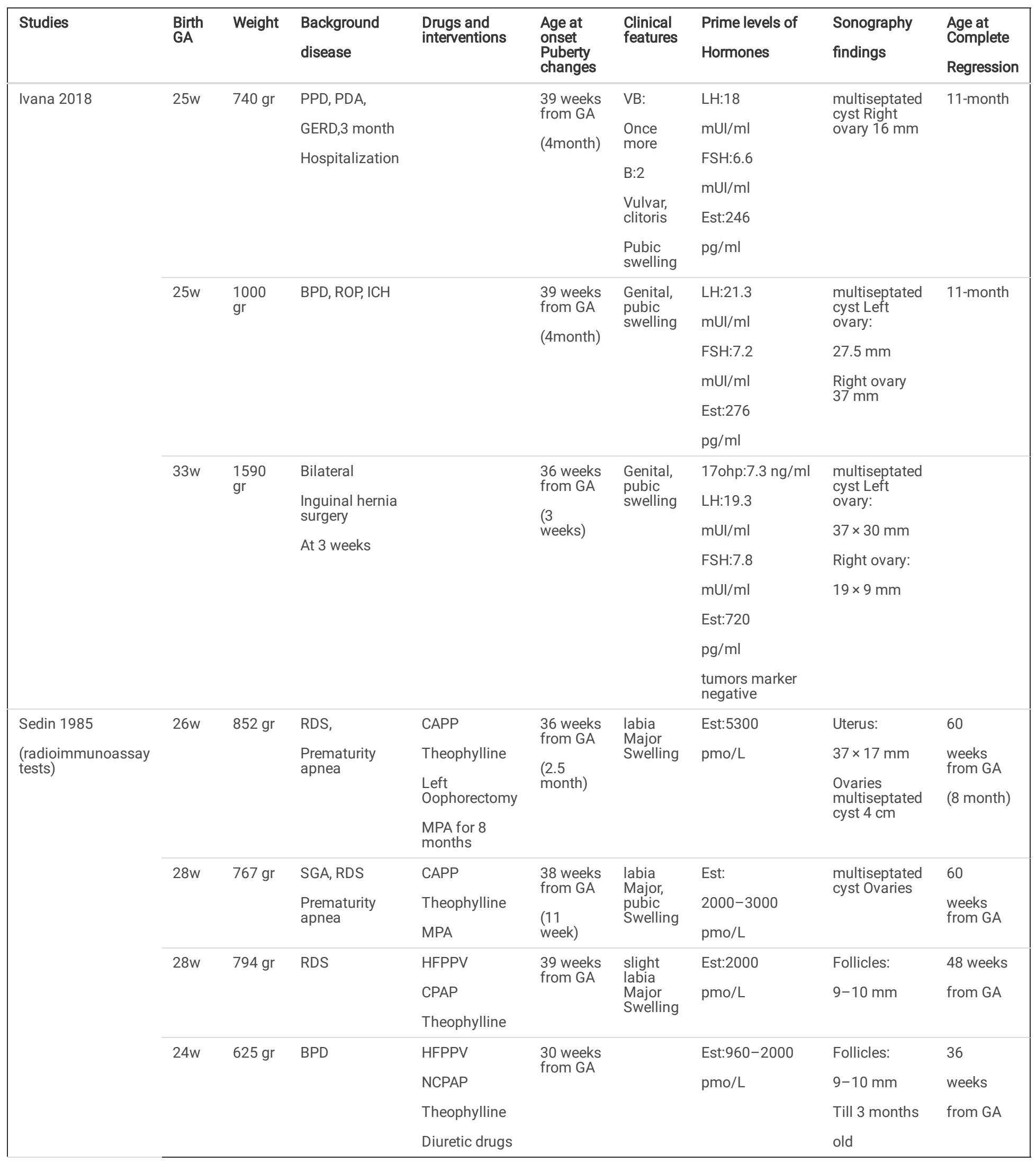

According to the regression of clinical presentation and para clinical data, Patient puberty continued to be monitored without prescribing any puberty prevention hormone medicines. After 4 months, breast size decreased to tanner stage 2 and vaginal bleeding was reported to have stopped reoccurrence. Reduction of puberty symptoms, laboratory improvements and sonography within a year of patient's observation reached the point that the Clinical symptoms returned to pre-puberty stage. The tests included these levels: LH 0.08, FSH 1.63, and Estradiol 8.3. Also, sonography reported the uterine size of $1.8 \mathrm{cc}$ and the ovaries volume of $0.9 \mathrm{cc}$ (Table 1$)$.

Fortunately, she can stand and walk with help and oxygen demand become limited to bedtime at the age of 16 months and 2 months later (at the age Loading [MathJax]/jax/output/CommonHTML/jax.js id not any oxygen therapy. Patient weight reached to $8600 \mathrm{gr}$ (10th percentile), the height to $78 \mathrm{~cm}$ (25th 
percentile) and the head circumference to $43.5 \mathrm{~cm}$. Thyroid function tests were under controlled with levothyroxine $25 \mathrm{mg} / \mathrm{daily}$ during one year. All of the above-mentioned symptoms could be interpreted by exaggerated mini puberty during infancy due to prematurity that shows the improvement in severe puberty symptoms without any extra treatments.

\section{Systematic Review}

Fourteen mini puberty cases from 8 studies were included in this systematic review. The mean preterm birth gestational age (GA) of infants with exaggerated mini puberty symptoms was 26.3 weeks, (S.D: 2.6 , range: $24-33$ weeks) and the mean birth weight was $963 \mathrm{~g}$ (S.D: 345 , range: 625-1590). Almost all of them have a history of long term hospitalization for prematurity problems (BPD) especially bronchopulmonary dysplasia and required long-term oxygen therapy. They also had retinopathy of prematurity (ROP), apnea prematurity, patent ductus arteriosus (PDA) and intracranial hemorrhage (ICH) (Table 2).

The mean onset of obvious signs of puberty in these infants is 39.28 weeks, (S.D: 4.5 , range: $30-48$ weeks) and on average, it was 3.5 months after birth (S.D: 1.4, range: 3 weeks to 6 months). A wide variety of sex maturity presentations are reported including: swelling labia major, thelarche, periodic vaginal bleeding and sometimes pubarche.

The age of termination of puberty symptoms is very diverse, which can be related to the severity of puberty, medical interventions or taken monitoring which was modified from about 2 weeks to 13 months (Table 2).

In this studies the wide variety of hormonal levels due to the severity of gonadotropin secretion and the subsequent stimulation of the ovaries and the enlargement of follicles secreting estradiol in the puberty level, can reflect the immaturity of the negative feedback mechanism in HPG axis in the premature infants. $[6,13]$ There were no significant correlation between hormones levels (LH, FSH and estradiol) with GA, weight at birth, age at onset puberty changes, and age at complete regression.

Of course, it is very important to note that in all the articles, the gradual decline of these hormonal changes strongly confirms that this pattern is a transient state. Interestingly, in patients undergoing therapeutic interventions, such as oophorectomy, GnRH agonist therapy, and medroxyprogesterone acetate (MPA), have shown that even after discontinuation of treatment, no signs of puberty is seen, which also confirms that the hormonal changes are temporary $[13,14$, 15]

Note that all symptoms have been considered and recorded according to the referrals of these patients, and they might have not been always reported due to the variety and severity of the symptoms and the lack of attention and knowledge of the parents or the routine examinations of infants by the doctors. Therefore, this clinical and para clinical (according to lab data and ultrasound findings) changes do not rare feature in VLBW preterm infants, and usually we neglect it. [13]

\section{Discussion}

During the first 2 years of life, high levels of gonadotropins intermittently to adult values have several roles in sexual development such as brain muscularization, longitudinal good testicular function (spermatogenesis), penile and testicular size, prostate growth in men and uterine and breast enlarge in girls and sebaceous gland, acne development and transient pubic hairs in both sexes $[5,16,17]$. In premature infants exaggerated mini puberty state are observed with severe puberty exchange in clinical, laboratory and ultrasonography data.

Serum levels of gonadotropins especially FSH in preterm infants are higher and more extended than term newborns particularly in girls. In both sex LH level ordinarily decrease by 6-9 months of age, but in premature infants is longer than one year $[4,6]$.

In the patient of this study periodic vaginal bleeding was seen same as Ivana, Gisselle, Bethany and Maria study $[6,15,18]$. That is not conventional event in mini puberty state in term infants. This undesirable state in the premature infants is not necessarily needs to use GnRH agonist. Although Gisselle [15] start leuprolide injections every 28 day and discontinued it at age of 2 as same as the Aefke study. However, after disconnection of hormonal therapy any signs of puberty did not develop and the initial diagnosis of central precocious puberty (CPP) was incorrect. [14]

Patient in this study has pubic hairs, Same selim study [6] and unlike Gisselle [15] and Maria study [18]. This symptom is consequent on gonadal and adrenals androgens secretion concomitant with the LH changes in the mini-Puberty [6].

By regression of puberty symptoms in the patient of this study during 12 months of monitoring, these were seen Respectively; disappearance of pubarche hair then stop vaginal bleeding after 4 months and complete evanesce of breast bud at 8th month of follow up.

The complete regression of puberty symptoms in our patient continue about 1 year due to severity of the prim presentation, although in the other studies have been reported from 2 weeks to 10 months due to less severity such as edema of the labia major. [13, 18, 19, 20,]

Altogether the severity of prematurity is not necessarily associated with the severity and variety of puberty presentations and the duration of symptoms.

Most of the patients in similar articles also had long-term hospitalizations because of bronchopulmonary dysplasia, recurrent prematurity apnea, primary pulmonary hypertension, and patent ductus arteriosus, retinopathy of prematurity and even intracranial hemorrhage which all problems are the causes of inflammatory mechanisms in the body. $[13,15,18,20]$

Synchronization of reduction in sex hormones and inflammatory markers (ferritin) may indicate that inflammatory process is an irritant marker for Loading [MathJax]/jax/output/CommonHTML/jax.js see the table). Likewise, in current study case, gradual regression of puberty symptoms concomitant to recovery 
of BPD symptoms and decrease to discontinuous of oxygen demand are observed.

The levels of hormones mentioned in several studies did not necessarily coincide with the severity of clinical symptoms and indicates other effective factors such as the method and accuracy of laboratory tests, different sensitivity of the receptors in the sexual organs in the patients, Medications (drugs used) and inflammatory factors due to the severity of chronic and systemic diseases. (see table 2)

There is not any cut of point in gonadotropins and sex hormones serum levels to distinguish mini puberty from central precocious puberty due to serious etiology such as tumors that needs GnRH agonist to be assessed. Thus Serum levels of gonadotropins and sex hormones are higher in premature infants than normal term infants with mini puberty.

Ultrasound data showed in mini puberty ovaries maturation are dominant to uterine size. In the patient of this study ovarian follicle size was over 10 mm is the opposite of selim study [6] and similar sedin, Ivana, mosallanejad, Gisselle, and Maria studies. [13,15,1819,20]

Despite the decrease of the sex hormone levels and reduction of uterine size and ovaries volume, follicles with the size of 9 mm are observed in sonography which shows the development process and atrophy of the follicles that is not necessarily related to puberty improvement, like Sedin's Study [13] result that can be remained till the age of 2 .

In Sedin's Study multiple ovarian cysts with an average diameter of $21 \mathrm{~mm}$ are found. In $50 \%$ of the cases reduction in the cysts are observed in the first month and in $25 \%$ of the cases in the second month. Also may persist at longer than three months in $10 \%$ of the cases [13].

If the cysts don't get smaller without clinical reduction, medroxyprogesterone acetate can be prescribed to decreasing the synthesis of ovarian estradiol [13].

However, it should be noted if regression of clinical symptoms are seen, medical treatment is not necessary because the enlargement and shrinkage of the follicles are seen naturally up to the age of 2. In Sedin's study Medroxyprogesterone has been suggested for treatment with estradiol above 2000 pmo/L (radioimmunoassay), which is not recommended because the fluctuating estradiol levels during the first 2 years of life is proportional to the change in the size of the follicles. [13]

Although treatment with medroxyprogesterone causes a faster reduction in estradiol levels, but the important sexual development roles of hormonal changes during the mini-puberty period, definitely minimal therapeutic intervention during this particular period is recommended. [13]

To prevent torsion, aspiration drainage is recommended in cysts that are larger than $4-5 \mathrm{~cm}$ [6]. However, the normal pathology of a $4 \mathrm{~cm}$ multiloculated cyst ovary in Sedin's patient who has undergone unilateral oophorectomy and then observation of enlarged ovarian cysts in the opposite ovaries which have been treated with medroxyprogesterone for some time, shows that should be avoided hasty surgery. Because the main pathology is not in the ovaries but it is caused by exaggerated increase in the secretion of gonadotropins during mini puberty due to prematurity, temporarily. So it is better to use exaggerated mini puberty word instead ovarian hyperstimulation syndrome in preterm infants [13]. Brain MRI with attention to Hypothalamus and pituitary is recommended to rule out the brain insult (tumor or anatomic disorders).

One of the premature infants in Sedin's study had no clinical signs of puberty. Only periodical tests showed increased levels of estradiol serum and about 10$\mathrm{mm}$ ovarian follicles till the age of 15 months which indicates the dynamic process of mini puberty even without clinical presentations. In a more comprehensive plane, with periodic monitoring such as hormonal testing, uterine and ovarian ultrasound findings, and attention to the clinical changes, it is possible to get a better understanding of the pattern of mini-pubertal changes and their importance in this critical period of life in the VLBW premature infants. [13].

It is recommended that after completing the evaluation of serious etiology for precocious puberty in preterm infancies, with careful monitoring, patients return to the pre-pubertal stage gradually, without any extra hormonal treatments.

\section{Conclusions}

Mini puberty is one of the most important events of infancy with intermittent changes puberty presentations and gives several interpretations for sexual development in both sexes. In premature infants exaggerated mini puberty is an intermittent state with severe puberty changes in clinical, laboratory and sonography data. The levels of gonadotropins in the preterm infants are higher and more extended than term newborns particularly in girls. Severity and association of puberty symptoms such as vaginal bleeding, the larche tanner above stage 2, pubic hair and also very high levels of gonadotropins, gonadal steroids and ovaries follicles size in preterm infants, is not enough to start treatments. It seems that paying attention to any puberty changes in very premature infants can help in better recognition of exaggerated mini puberty pattern during this important period of life.

\section{Abbreviations}

GA

gestational age

BPD

Bronchopulmonary dysplasia

HFPPV

High frequency positive pressure ventilation

Loading [MathJax]/jax/output/CommonHTML/jax.js 
Nasal continuous positive airway pressure

PDA

patent ductus arteriosus

$\mathrm{ICH}$

intracranial hemorrhage

Est

Estradiol serum

ROP

retinopathy of prematurity

$\mathrm{PPH}$

persistent pulmonary hypertension

DSD

Difference in Sex Development

MPA

medroxyprogesterone acetate

RDS

respiratory distress syndrome

SGA

Small for gestational age

ASD

Atrial septal defect

$\mathrm{CH}$

congenital hypothyroidism

VB

vaginal bleeding

B

breasts tanner stage

$\mathrm{PH}$

pubic hair tanner stage

$\mathrm{LH}$

Luteinizing hormone

$\mathrm{FSH}$

Follicle-stimulating hormone

VLBW

very low birth weight

\section{Declarations}

\section{Ethics approval and consent to participate}

As neonates are not capable of providing ethical consent to participate, the parents provided a written informed consent.

\section{Consent for publication}

The parents provided written informed consent for publication.

\section{Availability of data and materials}

The datasets used and analyzed during the current study are reported in the manuscript.

\section{Competing interests}

All authors declared no conflict of interest.

\section{Funding}

Not applicable.

\section{Authors' contributions}

Study conception and design: MAD, FG, GM and AAH. Manuscript preparation, supervision, administrative support and critical revision of the paper: MAD, FG, GM and AAH. All authors read and approved the final manuscript. 
The authors would like to thank the parent of patient who kindly provided us the photos to be published.

\section{References}

1. Rodolfo A. Mini-puberty and true puberty: Differences in testicular function. Ann Endocrinol. 2014 may;75:58-63. Doi:10.1016/j.ando.2014.03.001.

2. Kenneth C, Copeland CH, Steven CH. Mini-Puberty and Growth.Pediatrics 2016 June9; 138:(page number?) DOI: 10.1542/peds.2016-1301.

3. Maguelone GF, Anne MC, Jean AB.Evidence of testicular activity in early infancy. Journal of Clinical Endocrinology \& Metabolism 1973 July1; 37:148151 https://doi.org/10.1210/jcem-37-1-148.

4. Tanja Kuiri H, Ulla S, Leo D. Activation of the Hypothalamic Pituitary Gonadal Axis in Infancy:Minipuberty.Hormone reaserch in paediatrics 2014 july5;82:73-80 DOI: 10.1159/000362414.

5. Shlomo M, Richard JA, Allison BG, Ronald JK, Clifford JR. Williams Textbook of Endocrinology. United States: Elsevier; 2020.

6. Selim K, Osman B.Mini puberty and its interpretation.Turkish Pediatric Association 2014 May5; 49: 186-9D0I:10.5152/tpa.2014.2038.

7. Stoupa A, Samara D, Flechtner I, Pinto G, Jourdon I, González L, et al. Efficacy and safety of continuous subcutaneous infusion of recombinant human gonadotropins for congenital micropenis during early infancy. Horm Res Paediatr. 2017;87:103-10. doi:10.1159/0004.

8. Bonomi M, Vezzoli V, Krausz C, Guizzardi F, Vezzani S, Simoni M, et al. Italian Network on Central Hypogonadism. Characteristics of a nationwide cohort of patients presenting with isolated hypogonadotropic hypogonadism(IHH). Eur J Endocrinol. 2018;178:23-32. doi:10.1530/EJE-17-0065.

9. Lucia L, Marta C, Alberto L, Laura P, Susanna E.Up-To-Date Review About Minipuberty and Overview on Hypothalamic-Pituitary-Gonadal Axis Activation in Fetal and Neonatal Life. Front. Endocrinol 2018 July23;410 doi: 10.3389/fendo.2018.00410.

10. Quinton R, Mamoojee Y, Jayasena CN, Young J, Howard S, Dunkel L, et al. Society for Endocrinology UK guidance on the evaluation of suspected disorders of sexual development: emphasizing the opportunity to predict adolescent pubertal failure through a neonatal diagnosis of absent minipuberty. Clin Endocrinol. 2017;86:305-6. doi:10.1111/cen.13257.

11. Dwyer AA, Jayasena CN, Quinton R. Congenital hypogonadotropic hypogonadism: implications of absent mini-puberty.Minerva Endocrinol. (2016) 41:188-95.

12. Trine Holm J, Katharina Maria M, Marie Lindhardt L, Tina Kold J, Helle Raun A, Marianne Skovsager A, et al.Sex Differences in Reproductive Hormones During Mini-Puberty in Infants With Normal and Disordered Sex Development. The Journal of Clinical Endocrinology \& Metabolism 2018 june15;103: 3028-3037https://doi.org/10.1210/jc.2018-00482.

13. Sedin G, Bergquist C, Lindgren PG. Ovarian Hyperstimulation Syndrome in Preterm Infants. Pediatr Res. 1985;003:1-399818511.

14. Aafke HM, Gianni B. Vaginal bleeding in a 4-month-old preterm girl: extreme minipuberty mimicking central precocious puberty. Journal of pediatric 2011 DOI:10.1515/jpem.2011.363.

15. Gisselle P, Jenna K, Chimsom O. Jennifer L.Mini-Puberty of Infancy in Extreme Prematurity. J Pediatr Adolesc Gynecol. 2017;30:275-98.

16. Becker M, Oehler K, Partsch CJ, Ulmen U, Schmutzler R, Cammann H, et al. Hormonal "minipuberty" influences the somatic development of boys but not of girls up to the age of 6 years. Clin Endocrinol. 2015;83:694-701. doi:10.1111/cen.12827.

17. Mark AS, Pediatric E.Philadelphia: Elsevier; 2014(2).

18. Maria G, Michelle P, Sharon O, Craig A. Menstrual Bleeding as a Manifestation of Mini-Puberty of Infancy in Severe Prematurity. The Journal of pediatrics 2016 November;178: 292-295. doi:10.1016/j.jpeds.2016.08.021.

19. Mosallanejad A, et al. A Rare Case of Ovarian Hyperstimulation Syndrome in a Preterm Infant. Journal of Clinical Diagnostic Research. 2016 November;Vol. -10(11):D07-SD08.

20. Ivana V, Dragan K, Branka K, Melanija V, Ivana B, Sandra S. Preterm Ovarian Hyperstimulation Syndrome: A Clinical Presentation of Three Cases. Journal of Gynecology Neonatal Biology. 2018 June;18:ISSN: 2380-5595.

\section{Figures}



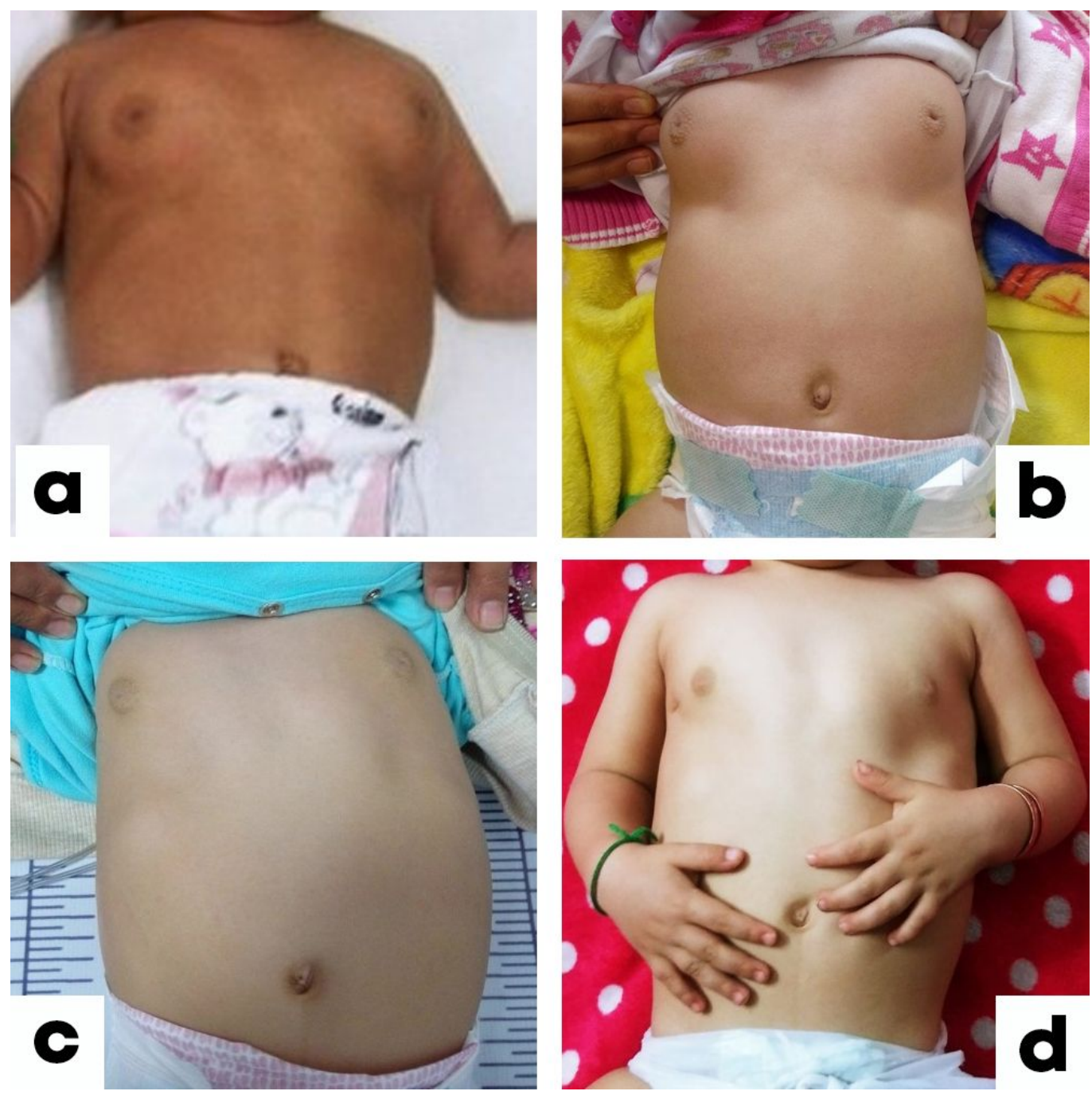

Figure 1

Notice the regression in the size of the breast during one year follow up. a) 6 months b) 9 months c) 13 months d) 18 months

\section{Supplementary Files}

This is a list of supplementary files associated with this preprint. Click to download.

- PRISMA2009checklist2.doc 